\title{
Lepton number violation at colliders from kinematically inaccessible gauge bosons
}

\author{
Richard Ruiz ${ }^{1,2, a_{(D)}}$ \\ ${ }^{1}$ Department of Physics, Institute for Particle Physics Phenomenology (IPPP), Durham University, Durham DH1 3LE, UK \\ 2 DARC, Green Lane, Durham DH1 3JU, UK
}

Received: 19 March 2017 / Accepted: 28 May 2017 / Published online: 6 June 2017

(C) The Author(s) 2017. This article is an open access publication

\begin{abstract}
We reevaluate the necessity of $W_{R}$ gauge bosons being kinematically accessible to test the left-right symmetric model (LRSM) at hadron colliders. In the limit that $W_{R}$ are too heavy, resonant production of sub-TeV Majorana neutri$\operatorname{nos} N$ can still proceed at the Large Hadron Collider (LHC) via the process $p p \rightarrow W_{R}^{ \pm *} \rightarrow N \ell^{ \pm} \rightarrow \ell^{ \pm} \ell^{ \pm}+n j$ if mediated by a far off-shell $W_{R}$. Traditional searches strategies are insensitive to this regime as they rely on momenta of final states scaling with $\mathrm{TeV}$-scale $M_{W_{R}}$. For such situations, the process is actually kinematically and topologically identical to the direct production (DP) process $p p \rightarrow$ $W_{\mathrm{SM}}^{ \pm *} \rightarrow N \ell^{ \pm} \rightarrow \ell^{ \pm} \ell^{ \pm}+n j$. In this context, we reinterpret $\sqrt{s}=8 \mathrm{TeV}$ LHC constraints on DP rates for the minimal LRSM. For $m_{N}=200-500 \mathrm{GeV}$ and right-left coupling ratio $\kappa_{R}=g_{R} / g_{L}$, we find $\left(M_{W_{R}} / \kappa_{R}\right)>1.1-1.8 \mathrm{TeV}$ at $95 \%$ CLs. Expected sensitivities to DP at 14 (100) TeV are also recast: with $\mathscr{L}=1(10) \mathrm{ab}^{-1}$, one can probe $\left(M_{W_{R}} / \kappa_{R}\right)<$ 7.9-8.9 (14-40) TeV for $m_{N}=100-700$ (1200) GeV, well beyond the anticipated sensitivity of resonant $W_{R}$ searches. Findings in terms of gauge invariant dimension-six operators with heavy $N$ are also reported.
\end{abstract}

\section{Introduction}

The left-right symmetric model (LRSM) [1-5] remains one of the best motivated high-energy completions of the Standard Model of Particle Physics (SM). It ties together the Majorana nature of neutrinos, their tiny masses in comparison to the electroweak (EW) scale $v_{\mathrm{EW}}$, and the chiral structure of EW interactions, seemingly disparate phenomena, to the simultaneous breakdown of $(B-L)$ conservation and left-right parity invariance at a scale $v_{R} \gg v_{\mathrm{EW}}$. Predicting a plethora of observations, the model is readily testable

\footnotetext{
a e-mail: richard.ruiz@durham.ac.uk
}

at current and near-future experiments; see [6-11] and the references therein.

At the Large Hadron Collider (LHC), searches [12,13] for $W_{R}$ gauge bosons and heavy Majorana neutrinos $N$, if kinematically accessible, focus on the well-studied, lepton number-violating $(\Delta L= \pm 2)$ Drell-Yan process [14],

$p p \rightarrow W_{R}^{ \pm} \rightarrow N \ell_{1}^{ \pm} \rightarrow \ell_{1}^{ \pm} \ell_{2}^{ \pm}+n j$.

As seen in Fig. 1a, Eq. (1) proceeds for $m_{N}<M_{W_{R}}$ first through the on-shell production of $W_{R}$, then by its decay to $N$. Recent investigations [16-20], however, have shown that one can obtain a considerable increase in sensitivity to the LRSM at colliders by relaxing the requisite charged lepton and jet multiplicities stipulated by Ref. [14] for Eq. (1) and similarly for the related single-top channel [21]. This is particularly true for $M_{W_{R}} \gg m_{N}, v_{\mathrm{EW}}$, which occurs naturally when $v_{R} \gtrsim \mathscr{O}(10) \mathrm{TeV}$ with neutrino triplet Yukawas $y^{\Delta_{R}} \lesssim$ $\mathscr{O}\left(10^{-2}\right)$. Incidentally, such scenarios are also favored by searches for flavor-changing neutral Higgs $(\mathrm{FCNH})$ transitions [22-25] and neutron EDMs [26,27]. Along these lines, we reevaluate the necessity of $W_{R}$ being kinematically accessible to test LR symmetry at hadron colliders.

In the limit that $M_{W_{R}}$ is of the order or above the total collider energy $\sqrt{s}$ but $m_{N} \ll \sqrt{s}$, Eq. (1) can still proceed if mediated instead by a far off-shell $W_{R}$. This is akin to the SM Fermi contact interaction. For $m_{N} \lesssim \mathscr{O}(1) \mathrm{TeV}, 8 \mathrm{TeV}$ searches $[12,13]$ for Eq. (1) are insensitive to this configuration due to the search premise itself: resonant $W_{R}$ production implies that momenta of final-state particles scale with $M_{W_{R}}$, justifying the use of $\mathrm{TeV}$-scale selection cuts in $[12,13]$. The choices of the cuts are motivated by limits from dijet searches that indicate $M_{W_{R}} \gtrsim 2.5 \mathrm{TeV}[28,29]$. Non-resonant $W_{R}$ mediation, however, implies that the partonic scale is naturally $\sqrt{\hat{s}} \sim m_{N} \lesssim \mathscr{O}(1) \mathrm{TeV}$, and therefore it is unlikely to lead to final states satisfying the kinematical criteria. For $m_{N} \gtrsim \mathscr{O}(1) \mathrm{TeV}$, present methods are sufficient [30]. 


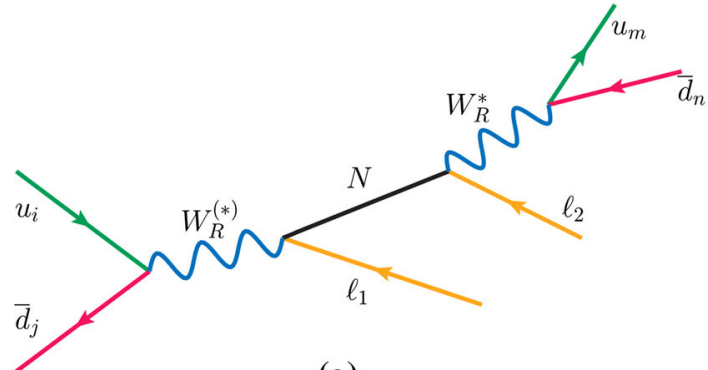

(a)

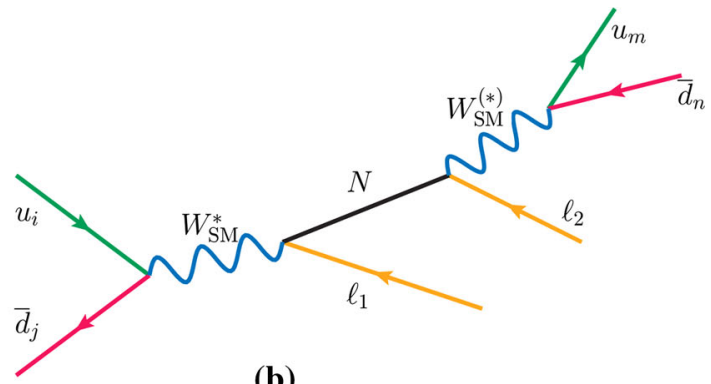

(b)

Fig. 1 Born diagrams for heavy Majorana $N$ production and decay via a $W_{R}$, b $W_{\mathrm{SM}}$ currents. Drawn using JaxoDraw [15]

Interestingly, while the underlying dynamics differ, for the $\left(M_{W_{R}}, m_{N}\right)$ range in consideration, the mass scale and topology of Eq. (1) are identical to the heavy Majorana neutrino direct production (DP) process

$p p \rightarrow W_{\mathrm{SM}}^{ \pm *} \rightarrow \ell_{1}^{ \pm} N \rightarrow \ell_{1}^{ \pm} \ell_{2}^{ \pm}+n j$.

As shown in Fig. 1b, this process, which may also be labeled as prompt production, transpires through off-shell SM $W$ bosons and occurs at the scale $m_{N}$ for $m_{N}>M_{W_{S M}}$ [3135]. Subsequently, hadron collider searches for Eq. (2) can be interpreted as searches for Eq. (1) in the $M_{W_{R}} \gtrsim \sqrt{s}$ limit. Moreover, despite its off-shell nature, the $W_{R}$ chiral couplings to quark and leptons remain encoded in azimuthal and polar distributions of the $\ell^{ \pm} \ell^{ \pm} n j$ system [36]. Thus, in principle, the dynamics of Eq. (1) can still be determined, even in mixed $W_{R}^{(*)}-W_{\mathrm{SM}}^{(*)}$ scenarios as considered in [3638]. It follows that this holds too for $e e / p p \rightarrow Z_{R}^{(*)} \rightarrow N N$

In the LRSM, heavy $N$ production can in principle also proceed through Eq. (2) and its neutral current equivalent via neutrino mixing. However, such mixing between left-handed flavor states $\ell$ and heavy mass eigenstate $N$, which scales as $V_{\ell N} \sim \sqrt{m_{v} / m_{N}}$, is necessarily small for the choice of $m_{N}$ in discussion and observed $m_{v}$. Subsequently, we neglect the contribution of Eq. (2) in the LRSM throughout this study. For further discussions, see, e.g., Refs. [37,39,40].

In this context, we reinterpret $\sqrt{s}=8 \mathrm{TeV}$ LHC limits on heavy Majorana neutrino DP cross sections [41,42] for the LRSM. For $m_{N}=200-500 \mathrm{GeV}$ and right-left coupling ratio $\kappa_{R}=g_{R} / g_{L}$, we find $\left(M_{W_{R}} / \kappa_{R}\right)<1.1-1.8$ $\mathrm{TeV}$ are excluded at $95 \%$ CLs. While weak, the limits are competitive with searches for resonant $M_{W_{R}}-N$ production $[13,30]$; however, for such low mass scales, the validity of this approach requires $\kappa_{R} \gg 1$. Projected sensitivities [43] to DP at the high-luminosity LHC and a hypothetical $100 \mathrm{TeV}$ Very Large Hadron Collider (VLHC) are recast into projections for the LRSM. At $14(100) \mathrm{TeV}$ and with $\mathscr{L}=1(10) \mathrm{ab}^{-1}$, one can probe $\left(M_{W_{R}} / \kappa_{R}\right)<7.9-$ $8.9(14-40) \mathrm{TeV}$ for $m_{N}=100-700$ (1200) GeV. We also translate sensitivity to $\left(M_{W_{R}} / \kappa_{R}\right)$ for coefficients of gauge invariant dimension-six operators in an effective field theory with right-handed neutrinos (NEFT) [44].

This study continues in the following order: in Sect. 2, the components of LRSM and NEFT relevant for this work are reviewed. We describe our methodology for reinterpreting (V)LHC limits in Sect. 3, and report results in Sect. 4. We summarize and conclude in Sect. 5.

\section{Theoretical framework}

We now briefly summarize the main relations of the minimal LRSM and NEFT relevant to this analysis.

\subsection{Minimal left-right symmetric model}

In the notation of [36], the $W_{R}$ quark chiral currents are

$\mathscr{L}_{W_{R}-q-q^{\prime}}=\frac{-\kappa_{R}^{q} g_{L}}{\sqrt{2}} \sum_{i, j=u, \ldots} \bar{u}_{i} V_{i j}^{R} W_{R \mu}^{+} \gamma^{\mu} P_{R} d_{j}+$ H.c.

Here, up-(down-)type quarks with flavor $i(j)$ are represented by $u_{i}\left(d_{j}\right) ; P_{R(L)}=\frac{1}{2}\left(1 \pm \gamma^{5}\right)$ is the right-hand (RH) [lefthand (LH)] chiral projection operator; $V_{i j}^{\mathrm{R}}$ denotes the $\mathrm{RH}$ analog of the Cabbibo-Kobayashi-Masakawa (CKM) matrix $V_{i j}^{\mathrm{L}}$; and $\kappa_{R}^{q} \in \mathbb{R}$ is an overall normalization for the $W_{R}$ interaction strength with respect to the SM weak coupling $g_{L}=\sqrt{4 \pi \alpha_{\mathrm{EM}}} / \sin \theta_{W}$. Despite nature maximally violating parity at low energies, $V_{i j}^{\mathrm{R}}$ retains its resemblance to $V_{i j}^{\mathrm{L}}$, with $\left|V_{i j}^{\mathrm{R}}\right|=\left|V_{i j}^{\mathrm{L}}\right|$ for generalized charge conjugation and $\left|V_{i j}^{\mathrm{R}}\right| \approx\left|V_{i j}^{\mathrm{L}}\right|+\mathscr{O}\left(m_{b} / m_{t}\right)$ for generalized parity [26,27,4547]. Throughout this study, we assume five massless quarks and, for simplicity, take $\left|V_{i j}^{L}\right|,\left|V_{i j}^{R}\right|$ to be diagonal with unit entries.

For leptonic coupling to $W_{R}$, we consider first the decomposition of neutrino chiral states $i, j$ into mass states $m, m^{\prime}$ : Assuming $i(m)=1, \ldots, 3, \mathrm{LH}$ (light) states and $j\left(m^{\prime}\right)=$ $1, \ldots, n, \mathrm{RH}$ (heavy) states, we can relate the chiral neutrino states and mass eigenstates by the rotation 


$$
\left(\begin{array}{c}
v_{L i} \\
N_{R j}^{c}
\end{array}\right)=\left(\begin{array}{cc}
U_{3 \times 3} & V_{3 \times n} \\
X_{n \times 3} & Y_{n \times n}
\end{array}\right)\left(\begin{array}{c}
v_{m} \\
N_{m^{\prime}}^{c}
\end{array}\right)
$$

Without the loss of generality, we take the rotation of the charged leptons into the mass basis as the identity. The $U_{3 \times 3}$ component of Eq. (3) is then recognized as the observed light neutrino mixing matrix. In analogy to $U_{\ell m}$, the entry $Y_{\ell m^{\prime}}\left(X_{\ell m}\right)$ quantifies the mixing between the heavy (light) mass state $N_{m^{\prime}}\left(v_{m}\right)$ and the RH chiral state with corresponding flavor $\ell$. Hence, the mixing entries scale as $\left|Y_{\ell m^{\prime}}\right|^{2} \sim$ $\mathscr{O}(1)$ and $\left|X_{\ell m}\right|^{2} \sim 1-\left|Y_{\ell m^{\prime}}\right|^{2} \sim \mathscr{O}\left(m_{v_{m}} / m_{N_{m^{\prime}}}\right)$ [14]. Explicitly, the RH flavor state $N_{\ell}$ in the mass basis is then $[35,36]$

$$
N_{\ell}=\sum_{m=1}^{3} X_{\ell m} v_{m}^{c}+\sum_{m^{\prime}=1}^{n} Y_{\ell m^{\prime}} N_{m^{\prime}}
$$

With this, the $W_{R}$ chiral currents for leptons are $[35,36]$

$$
\begin{aligned}
\mathscr{L}_{W_{R}-\ell-v / N}= & \frac{-\kappa_{R}^{\ell} g_{L}}{\sqrt{2}} \sum_{\ell=e}^{\tau} \overline{N_{\ell}} W_{R \mu}^{+} \gamma^{\mu} P_{R} \ell^{-}+\text {H.c. } \\
= & \frac{-\kappa_{R}^{\ell} g_{L}}{\sqrt{2}} \sum_{\ell=e}^{\tau}\left[\sum_{m=1}^{3} \overline{v_{m}^{c}} X_{\ell m}^{\dagger}+\sum_{m^{\prime}=1}^{3} \overline{N_{m^{\prime}}} Y_{\ell m^{\prime}}^{\dagger}\right] \\
& \times W_{R \mu}^{+} \gamma^{\mu} P_{R} \ell^{-}+\text {H.c. }
\end{aligned}
$$

As for quarks, $\kappa_{R}^{\ell} \in \mathbb{R}$ normalizes the $W_{R}$ coupling to leptons. Throughout this analysis, we adopt the conventional benchmark scenario and consider only the lightest heavy neutrino mass state $N_{m^{\prime}=1}$, which we denote $N$.

\subsection{Effective field theory with heavy neutrinos}

Heavy neutrino effective field theory (NEFT) $[44,48,49]$ is a powerful extension of the SM EFT $[50,51]$ that allows for a consistent and agnostic parameterization of new, high-scale, weakly coupled physics when $N$ mass scales comparable to $v_{\text {EW }}$. As TeV-scale $L$ violation implies $[52,53]$ the existence of a particle spectrum beyond the canonical Type I seesaw [54-57], it is natural to consider DP sensitivities in terms of NEFT operators.

After extending the SM by three $N_{R}$, the most general renormalizable theory that can be constructed from SM symmetries is the Type I seesaw Lagrangian,

$\mathscr{L}_{\text {Type I }}=\mathscr{L}_{\text {SM }}+\mathscr{L}_{N \text { Kin.+Mass }}+\mathscr{L}_{N \text { Yukawa }}$.

Respectively, the three terms are the SM Lagrangian, the kinetic and Majorana mass terms for $N_{R}$, and the Yukawa couplings responsible for Dirac neutrino masses. From this, the NEFT Lagrangian can be built by further extending $\mathscr{L}_{\text {Type I before EW symmetry breaking (EWSB) by all SU(3) }}$ $\otimes \mathrm{SU}(2)_{L} \otimes \mathrm{U}(1)_{Y}$-invariant, irrelevant (mass dimension $d>4$ ) operators containing Type I seesaw fields:

$\mathscr{L}_{\text {NEFT }}=\mathscr{L}_{\text {Type I }}+\sum_{d=5} \sum_{i} \frac{\alpha_{i}}{\Lambda^{(d-4)}} \mathscr{O}_{i}^{(d)}$.

Here, $\alpha_{i}<\mathscr{O}(4 \pi)$ are dimensionless coupling coefficients, $\Lambda \gg \sqrt{\hat{s}}$ is the mass scale of the underlying theory, and $\mathscr{O}_{i}^{(d)}$ are gauge invariant permutations of Type I field operators. The list of $\mathscr{O}_{i}^{(d)}$ are known explicitly for $d=5$ [48], 6 [44], and 7 [49], and can be built for larger $d$ following [58,59].

At $d=6$, the four-fermion $\mathscr{O}_{i}^{(6)}$ giving rise to the same parametric dependence on $m_{N}$ in the partonic cross section $\hat{\sigma}$ as both DP and the LRSM for $M_{W_{R}} \gg \sqrt{\hat{s}}$ are

$\mathscr{O}_{V}^{(6)}=\left(\bar{d} \gamma^{\mu} P_{R} u\right)\left(\bar{e} \gamma_{\mu} P_{R} N_{R}\right)$ and

$\mathscr{O}_{S 3}^{(6)}=\left(\bar{Q} \gamma^{\mu} P_{R} N_{R}\right) \varepsilon\left(\bar{L} \gamma_{\mu} P_{R} d\right)$.

In Eq. (7), $\varepsilon$ is the totally antisymmetric tensor. After EWSB and decomposing $N_{R}$ according to Eq. (4), but neglecting $\mathscr{O}\left(X_{\ell m}\right)$ terms, the operators become

$\mathscr{O}_{V}^{(6)}=\sum_{m^{\prime}=1}\left(\bar{d} \gamma^{\mu} P_{R} u\right)\left(\bar{\ell} \gamma_{\mu} P_{R} Y_{\ell m^{\prime}} N_{m^{\prime}}\right)$ and
$\mathscr{O}_{S 3}^{(6)}=\sum_{m^{\prime}=1}\left(\bar{Q} \gamma^{\mu} P_{R} Y_{\ell m^{\prime}} N_{m^{\prime}}\right)\left(\bar{\ell} \gamma_{\mu} P_{R} d\right)$.

As in the LRSM case, we consider only the $N_{m^{\prime}=1}$ state with mixing as given in Eqs. (40), (41).

\section{Mimicking direction production with left-right symmetry}

In this section we describe our procedure for extracting bounds on LRSM and NEFT quantities from observed and expected (V)LHC limits on heavy Majorana neutrino DP rates. Our computational setup is summarized in Sect. (3.1). We start by constructing the observable $\varepsilon\left(M_{W_{R}}\right)$, which we will ultimately constrain.

The Born-level, partonic heavy $N$ production cross section via (on- or off-shell) $W_{R}$ currents,

$q_{1} \overline{q_{2}} \rightarrow W_{R}^{ \pm(*)} \rightarrow N \ell_{1}^{ \pm}$

with arbitrary lepton mixing is given generically by [36]

$\frac{\mathrm{d} \hat{\sigma}^{\text {LRSM }}}{\mathrm{d} \Omega_{\ell}}=\frac{3 \hat{\sigma}_{\text {Tot. }}^{\text {LRSM }}}{2^{3} \pi\left(2+r_{N}\right)}\left[\left(1-\cos \theta_{\ell}\right)^{2}+r_{N} \sin ^{2} \theta_{\ell}\right]$

where $r_{N} \equiv m_{N}^{2} / \hat{s}$ and the total cross section is

$$
\begin{aligned}
\hat{\sigma}_{\text {Tot. }}^{\text {LRSM }} & =\frac{\kappa_{R}^{q 2} \kappa_{R}^{\ell 2} g_{L}^{4}}{2^{7} 3 N_{c} \pi} \frac{\left|Y_{\ell N}\right|^{2} \hat{s}\left(1-r_{N}\right)^{2}\left(2+r_{N}\right)}{\left[\left(\hat{s}-M_{W_{R}}^{2}\right)^{2}+\left(M_{W_{R}} \Gamma_{W_{R}}\right)^{2}\right]} \\
& \approx \frac{\kappa_{R}^{q 2} \kappa_{R}^{\ell 2} g_{L}^{4}}{2^{7} 3 N_{c} \pi}\left|Y_{\ell N}\right|^{2} \frac{\hat{s}}{M_{W_{R}}^{4}}\left(1-r_{N}\right)^{2}\left(2+r_{N}\right) .
\end{aligned}
$$


In the last line we take the $M_{W_{R}} \gg \sqrt{\hat{s}}$ limit. For DP, the analogous partonic cross section is

$\frac{\mathrm{d} \hat{\sigma}^{\mathrm{DP}}}{\mathrm{d} \Omega_{\ell}}=\frac{3 \hat{\sigma}_{\mathrm{Tot}}^{\mathrm{DP}}}{2^{3} \pi\left(2+r_{N}\right)}\left[\left(1-\cos \theta_{\ell}\right)^{2}+r_{N} \sin ^{2} \theta_{\ell}\right]$

where the total partonic rate for $\sqrt{\hat{s}} \gg M_{W_{\mathrm{SM}}}$ is, similarly,

$$
\begin{aligned}
\hat{\sigma}_{\text {Tot. }}^{\text {DP }} & =\frac{g_{L}^{4}}{2^{7} 3 N_{c} \pi} \frac{\left|V_{\ell N}\right|^{2} \hat{s}\left(1-r_{N}\right)^{2}\left(2+r_{N}\right)}{\left[\left(\hat{s}-M_{W_{R}}^{2}\right)^{2}+\left(M_{W} \Gamma_{W}\right)^{2}\right]} \\
& \approx \frac{g_{L}^{4}\left|V_{\ell N}\right|^{2}}{2^{7} 3 N_{c} \pi} \frac{1}{\hat{s}}\left(1-r_{N}\right)^{2}\left(2+r_{N}\right) .
\end{aligned}
$$

Comparing the differential and integrated expressions one sees crucially that the angular and $m_{N}$ dependence in the two processes are the same. This follows from the maximally parity violating $V \pm A$ structures of the $W_{\mathrm{SM}} / W_{R}$ couplings. Naïvely, one expects the orthogonal chiral couplings to invert the leptons' polarizations with respect to the mediator. However, as the mediators' polarizations are also relatively flipped with respect to the initial-state quarks, the outgoing lepton polarization with respect to initial-state quarks, i.e., $\cos \theta_{\ell}$, is the same. Hence, universality of $W_{R}$ chiral couplings to quarks and leptons in the LRSM can be tested without resonantly producing it. The precise handedness of the couplings can be inferred from azimuthal and polar distributions of the $\ell^{ \pm} \ell^{ \pm} j j$ final state [36] as well as single-top channel [60]. As DP searches do not (and should not) rely on forwardbackward cuts, which are sensitive to parity asymmetries, their reinterpretation in terms of the LRSM for non-resonant $W_{R}$ is justified.

Branching rates of $N$ to a final state $A$ can be expressed in terms of the calculable $N \rightarrow A$ partial widths,

$\mathrm{BR}(N \rightarrow A) \equiv \frac{\Gamma(N \rightarrow A)}{\sum_{i} \Gamma\left(N \rightarrow A_{i}\right)}$.

For $M_{W_{R}} \gg m_{N}$, the $M_{W_{R}}$ dependence in Eq. (16) cancels. Hence, the Born-level, partonic same-sign lepton cross section in the LRSM,

$q_{1} \overline{q_{2}} \rightarrow W_{R}^{ \pm *} \rightarrow N \ell_{1}^{ \pm} \rightarrow \ell_{1}^{ \pm} \ell_{2}^{ \pm} X$,

under the narrow width approximation for $N$ is

$$
\begin{aligned}
& \hat{\sigma}\left(q_{1} \overline{q_{2}} \rightarrow N \ell_{1}^{ \pm} \rightarrow \ell_{1}^{ \pm} \ell_{2}^{ \pm} X\right) \\
& \quad \approx \hat{\sigma}_{\text {Tot. }}^{\mathrm{LRSM}} \times \operatorname{BR}\left(N \rightarrow \ell_{2}^{ \pm} X\right) \\
& \equiv \varepsilon^{\ell_{1} \ell_{2}}\left(M_{W_{R}}\right) \times \hat{\sigma} .
\end{aligned}
$$

In the last line we collect LRSM parameters into the single, dimensionful $\left(\mathrm{TeV}^{-4}\right)$ coefficient

$\varepsilon^{\ell_{1} \ell_{2}}\left(M_{W_{R}}\right)=\frac{\kappa_{R}^{q 2} \kappa_{R}^{\ell 2}}{M_{W_{R}}^{4}}\left|Y_{\ell_{1} N}\right|^{2} \operatorname{BR}\left(N \rightarrow \ell_{2}^{ \pm} q_{1}^{\prime} \overline{q_{2}^{\prime}}\right)$.
The "reduced" partonic cross section $\tilde{\hat{\sigma}}$ contains all kinematical and $m_{N}$ dependence that must be convolved with parton distribution functions (PDFs) to build the hadronic cross section. For the $e^{ \pm} \mu^{ \pm}$mixed-flavor state, a summation over $\varepsilon^{e \mu}$ and $\varepsilon^{\mu e}$ is implied.

Inclusive, hadronic level cross sections are obtained from the Collinear Factorization Theorem,

$$
\begin{aligned}
& \sigma(p p \rightarrow A+X)=f \otimes f \otimes \hat{\sigma} \\
& =\frac{1}{\delta_{i j}+1} \sum_{i, j=u, g, \ldots} \int_{\tau_{0}}^{1} \mathrm{~d} \xi_{1} \int_{\tau_{0} / \xi_{1}}^{1} \mathrm{~d} \xi_{2} \\
& \quad \times\left[f_{i / p}\left(\xi_{1}, \mu_{f}\right) f_{j / p}\left(\xi_{2}, \mu_{f}\right)+(1 \leftrightarrow 2)\right] \hat{\sigma}(i j \rightarrow A) .
\end{aligned}
$$

It expresses the production rate of $A$ (and arbitrary beam remnant $X$ ) in $p p$ collisions as the convolution $(\otimes)$ of the $i j \rightarrow A$ partonic process rate and the process-independent PDFs $f_{k / p}\left(\xi, \mu_{f}\right)$, which for parton species $k$ with longitudinal momentum $p_{z}=\xi E_{p}$ resums collinear splittings up to the scale $\mu_{f}$. The kinematic threshold $\tau_{0}$ is the scale below which the process is kinematically forbidden. For heavy $N$ production, $\tau_{0}=m_{N}^{2} / s$. In terms of $\varepsilon\left(M_{W_{R}}\right)$, the hadronic equivalent of Eq. (19) is

$\sigma\left(p p \rightarrow N \ell_{1}^{ \pm} \rightarrow \ell_{1}^{ \pm} \ell_{2}^{ \pm}+X\right)=\varepsilon\left(M_{W_{R}}\right) \times \tilde{\sigma}$.

Here, $\tilde{\sigma}$ is the "reduced" hadronic cross section and is related to $\tilde{\hat{\sigma}}$ by the convolutions $\tilde{\sigma}=f \otimes f \otimes \tilde{\hat{\sigma}}$. As the nextto-leading order (NLO) in QCD corrections for arbitrary DY processes largely factorize from the hard scattering process [61,62], Eq. (23) holds at NLO:

$\sigma^{\mathrm{NLO}}\left(p p \rightarrow N \ell_{1}^{ \pm} \rightarrow \ell_{1}^{ \pm} \ell_{2}^{ \pm}+X\right)=\varepsilon\left(M_{W_{R}}\right) \times \sigma^{\tilde{\mathrm{NLO}}}$.

Premising that reported LHC limits on the DP cross section can be applied to the LRSM for kinematically inaccessible $W_{R}$, Eq. (24) shows how to translate the upper bound on the rate into an upper bound on $\varepsilon\left(M_{W_{R}}\right)$.

For the NEFT operators in Eq. (7), the corresponding partonic scattering rates are given by [44]

$$
\begin{aligned}
& \hat{\sigma}_{S 3}\left(u \bar{d} \rightarrow N \ell_{1}^{ \pm} \rightarrow \ell_{1}^{ \pm} \ell_{2}^{ \pm} X\right)=\frac{\alpha_{S 3}^{2}\left|Y_{N} \ell_{1}\right|^{2}}{2^{7} 3 N_{c} \pi} \frac{\hat{s}}{\Lambda^{4}} \\
& \times\left(1-r_{N}\right)^{2}\left(2+r_{N}\right) \times \operatorname{BR}\left(N \rightarrow \ell_{2} X\right), \\
& \hat{\sigma}_{V}\left(u \bar{d} \rightarrow N \ell_{1}^{ \pm} \rightarrow \ell_{1}^{ \pm} \ell_{2}^{ \pm} X\right)=\frac{4 \alpha_{V}^{2}}{\alpha_{S 3}^{2}} \hat{\sigma}_{S 3} .
\end{aligned}
$$

Comparing with Eqs. (10)-(13), one finds the mapping

$\mathscr{O}_{S 3}^{(6)}: \varepsilon^{\ell_{1} \ell_{2}}\left(M_{W_{R}}\right)=\frac{\alpha_{S 3}^{2}}{\Lambda^{4}}\left|Y_{N} \ell_{1}\right|^{2} \operatorname{BR}\left(N \rightarrow \ell_{2} X\right)$,
$\mathscr{O}_{V}^{(6)}: \varepsilon^{\ell_{1} \ell_{2}}\left(M_{W_{R}}\right)=\frac{4 \alpha_{V}^{2}}{\Lambda^{4}}\left|Y_{N} \ell_{1}\right|^{2} \operatorname{BR}\left(N \rightarrow \ell_{2} X\right)$.

This allows the further interpretation of $\varepsilon\left(M_{W_{R}}\right)$. 


\subsection{Computational setup}

Practically speaking, the NLO-accurate reduced cross section is determined using the FeynRules-based [63-65] NLO-accurate Effective Left-Right Symmetric Model file of [20] and MadGraph5_amc@NLO [66]. The process

$p p \rightarrow W_{R}^{ \pm *} \rightarrow N \mu^{ \pm}+X$

is calculated at NLO accuracy assuming test inputs:

$$
\begin{aligned}
& \left\{M_{\text {Test }}\right\}: M_{W_{R}}=200 \mathrm{TeV}, \quad \kappa_{R}^{\ell, q}=1, \\
& \left|Y_{\mu N}\right|=1, \quad \operatorname{BR}(N \rightarrow \mu X)=1 .
\end{aligned}
$$

For the choice of EW inputs, PDFs, etc., we follow Ref. [20]. Denoting the $\varepsilon\left(M_{W_{R}}\right)$ corresponding to the Eq. (30) as $\varepsilon\left(M_{\text {Test }}\right), \tilde{\sigma}^{\mathrm{NLO}}$ is obtained from the relationship

$\tilde{\sigma}^{\mathrm{NLO}}=\frac{\sigma^{\mathrm{NLO}}\left(p p \rightarrow N \mu^{ \pm}+X ;\left\{M_{\text {Test }}\right\}\right)}{\varepsilon\left(M_{\text {Test }}\right)}$.

\section{Results and discussion}

We now report the observed sensitivity to the LRSM from DP searches in the $\mu \mu / e e / e \mu$ channels by the CMS experiment at $\sqrt{s}=8 \mathrm{TeV}$ with $\mathscr{L}=19.7 \mathrm{fb}^{-1}$ [41,42]. We also report expected sensitivities based on $14 \mathrm{TeV}$ projections with $\mathscr{L}=100 \mathrm{fb}^{-1}$ and $1 \mathrm{ab}^{-1}$ [43], as well as at $100 \mathrm{TeV}$ with $\mathscr{L}=10 \mathrm{ab}^{-1}$ [43]. In all cases, 95\% confidence level (CL) limits are obtained/reproduced via the $\mathrm{CL}_{s}$ method [6769], using the information available in [41-43], and assuming Poisson distributions for signal and background processes. After obtaining the expected (observed) DP cross section limits $\sigma_{\text {Exp. (Obs.) }}^{95 \% \mathrm{CL}_{s}}$, LRSM constraints are determined from the "reduced" cross section $\tilde{\sigma}$, as defined in Eq. (31), with the relation

$\varepsilon_{\text {Exp. (Obs.) }}^{\ell_{1} \ell_{2}}\left(M_{W_{R}}\right)=\frac{\sigma_{\text {Exp. (Obs.) }}^{95 \% \mathrm{CL}_{s}}}{\tilde{\sigma}^{\mathrm{NLO}}}$.

In Fig. 2 we plot as a function of $m_{N}$ the $8 \mathrm{TeV}$ CMS upper bounds on $\varepsilon\left(M_{W_{R}}\right)$ for the (a) $\mu \mu$ (dash-dot) as well as (b) $e \mu$ (dash-dot) and $e e$ (upside-down triangle) channels. One finds comparable limits for all modes, with

$\mu^{ \pm} \mu^{ \pm}: \varepsilon^{\ell \ell}\left(M_{W_{R}}\right) \lesssim 0.05 \mathrm{TeV}^{-4}$,

$e^{ \pm} \mu^{ \pm}, e^{ \pm} e^{ \pm}: \varepsilon^{\ell \ell}\left(M_{W_{R}}\right) \lesssim 0.1 \mathrm{TeV}^{-4}$.

For $m_{N} \lesssim 150 \mathrm{GeV}, W_{\mathrm{SM}}$ production greatly diminishes sensitivity. A weaker limit for $e$-based channels is due to the larger fake and charge misidentification rates for electrons than for muons, particularly from top quarks. These features are seen consistently in projections.
In Fig. 2a, the expected sensitivity to $\varepsilon^{\mu \mu}\left(M_{W_{R}}\right)$ at $14 \mathrm{TeV}$ with $\mathscr{L}=100 \mathrm{fb}^{-1}$ (solid-triangle) and $1 \mathrm{ab}^{-1}$ (dash-dotdiamond) are shown. We find that, for $m_{N}=100-700 \mathrm{GeV}$, one can potentially exclude:

$$
\begin{aligned}
& \mathscr{L}_{100 \mathrm{fb}^{-1}}^{14 \mathrm{TeV}}: \varepsilon^{\mu \mu}\left(M_{W_{R}}\right) \lesssim 5 \times 10^{-4} \mathrm{TeV}^{-4}, \\
& \mathscr{L}_{1 \mathrm{ab}^{-1}}^{14 \mathrm{TeV}}: \varepsilon^{\mu \mu}\left(M_{W_{R}}\right) \lesssim 9 \times 10^{-5} \mathrm{TeV}^{-4}
\end{aligned}
$$

At a future $100 \mathrm{TeV}$ VLHC, the large increase in parton density coupled with proposed integrated luminosity goals of $10-20 \mathrm{ab}^{-1}$ [70] implies a considerable jump in sensitivity to $\varepsilon\left(M_{W_{R}}\right)$ for EW-scale $N$. For $m_{N}=100-1200 \mathrm{GeV}$, the $\mu \mu$ (dot-star) in Fig. 2a) and $e \mu$ (dot-star) in Fig. 2b) final state can probe with $10 \mathrm{ab}^{-1}$ :

$\varepsilon^{\mu \mu}\left(M_{W_{R}}\right) \lesssim 2 \times 10^{-7}-1 \times 10^{-6} \mathrm{TeV}^{-4}$,

$\varepsilon^{e \mu}\left(M_{W_{R}}\right) \lesssim 2 \times 10^{-7}-7 \times 10^{-6} \mathrm{TeV}^{-4}$.

Derived limits on $\varepsilon\left(M_{W_{R}}\right)$ hold for rather generic LR scenarios. Under the strong (but typical) assumptions of a minimal LRSM setting, we can rewrite constraints as lower bounds on the ratio of $M_{W_{R}}$ and $\kappa_{R}^{q, \ell}$. Specifically, assuming gauge coupling universality, one has

$\kappa_{R} \equiv \kappa_{R}^{q}=\kappa_{R}^{\ell}$.

For single flavor final states, we take the aligned lepton mixing limit Eq. (40), whereas for the mixed flavor channel, we take the maximally mixed limit Eq. (41), i.e.,

$\left|Y_{\ell N}\right| \approx 1$ and $\operatorname{BR}\left(N \rightarrow \ell^{ \pm} X\right) \approx 1$, or

$\left|Y_{e N}\right| \approx\left|Y_{\mu N}\right| \approx 1 / \sqrt{2}$ and

$\operatorname{BR}\left(N \rightarrow e^{ \pm} X\right) \approx \operatorname{BR}\left(N \rightarrow \mu^{ \pm} X\right) \approx 1 / 2$.

While $N$ can decay with equal likelihood to $\ell_{i}^{+}$and $\ell_{i}^{-}$, the same-sign charge stipulation reduces the effective branching by $1 / 2$. With this, we invert $\varepsilon\left(M_{W_{R}}\right)$, giving

$\frac{M_{W_{R}}}{\kappa_{R}}=\frac{1}{\sqrt[4]{\eta \times \varepsilon^{\ell_{1} \ell_{2}}\left(M_{W_{R}}\right)}}, \quad \eta=\left\{\begin{array}{l}2, \ell_{1}=\ell_{2} \\ 4, \ell_{1} \neq \ell_{2}\end{array}\right.$,

where $\eta$ accounts for charge and flavor multiplicities.

In Fig. 2c, d, respectively, we show the lower bounds on $\left(M_{W_{R}} / \kappa_{R}\right)$ for the same configurations as (a) and (b). For all channels, the observed $8 \mathrm{TeV}$ limits span:

$m_{N}=100-200 \mathrm{GeV}:\left(\frac{M_{W_{R}}}{\kappa_{R}}\right) \gtrsim 0.7-1.8 \mathrm{TeV}$,

$m_{N}=200-700 \mathrm{GeV}:\left(\frac{M_{W_{R}}}{\kappa_{R}}\right) \gtrsim 1.1-1.8 \mathrm{TeV}$.

At $\sqrt{s}=14 \mathrm{TeV}$ with $\mathscr{L}=100 \mathrm{fb}^{-1}$ and $1 \mathrm{ab}^{-1}$, the $\mu \mu$ final state can exclude for $m_{N}=100-700 \mathrm{GeV}$ : 


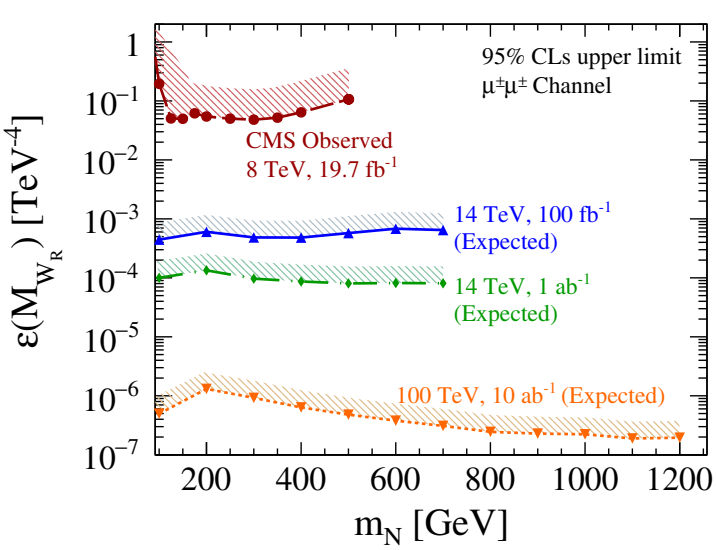

(a)

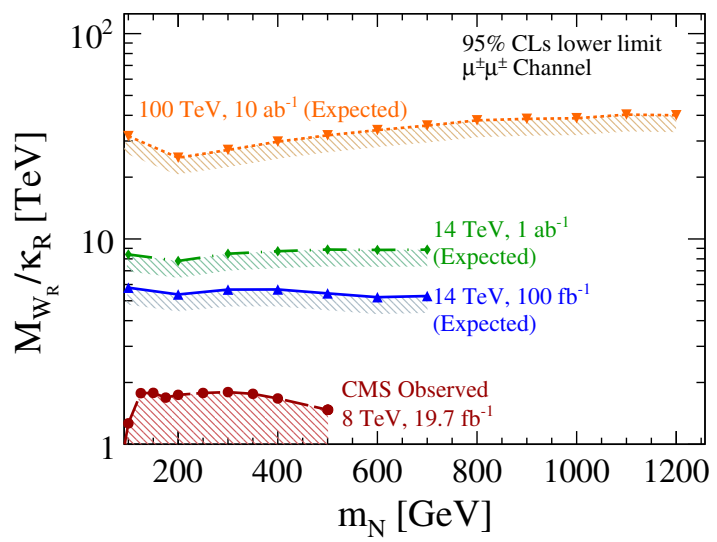

(c)

Fig. 2 a As a function of $m_{N}$, observed $8 \mathrm{TeV}$ LHC upper bound on $\varepsilon^{\mu \mu}\left(M_{W_{R}}\right)($ dash-dot $)$, expected $14 \mathrm{TeV}$ sensitivity with $\mathscr{L}=100 \mathrm{fb}^{-1}$ (solid-triangle) and $1 \mathrm{ab}^{-1}$ (dash-dot-diamond), and expected $100 \mathrm{TeV}$ VLHC sensitivity with $10 \mathrm{ab}^{-1}$ (dot-star). b Same as a but with $e^{ \pm} \mu^{ \pm}$

$\mathscr{L}_{100 \mathrm{fb}^{-1}}^{14 \mathrm{TeV}}:\left(\frac{M_{W_{R}}}{\kappa_{R}}\right) \lesssim 5.2-5.8 \mathrm{TeV}$,

$\mathscr{L}_{1 \mathrm{ab}^{-1}}^{14 \mathrm{TeV}}:\left(\frac{M_{W_{R}}}{\kappa_{R}}\right) \lesssim 7.8-8.9 \mathrm{TeV}$.

Comparable sensitivity in the $e e$ and $e \mu$ channels is expected. At $100 \mathrm{TeV}$ with $10 \mathrm{ab}^{-1}$, the $\mu \mu$ and $e \mu$ channels for $m_{N}=100-1200 \mathrm{GeV}$ are sensitive to

$\mu^{ \pm} \mu^{ \pm}:\left(\frac{M_{W_{R}}}{\kappa_{R}}\right) \lesssim 25-40 \mathrm{TeV}$,

$e^{ \pm} \mu^{ \pm}:\left(\frac{M_{W_{R}}}{\kappa_{R}}\right) \lesssim 14-33 \mathrm{TeV}$.

We note that the sharp cutoffs at $m_{N}=500,700$, and 1200 $\mathrm{GeV}$ for the several scenarios in Fig. $3 \mathrm{a}$ are due to the limited number of mass hypotheses considered in [41-43]. A dedicated analysis would show sensitivity to larger $m_{N}$.

To compare with searches for resonant $W_{R}-N$ production, we plot in Fig. 3a the region of the $\left(M_{W_{R}}, m_{N}\right)$ param-

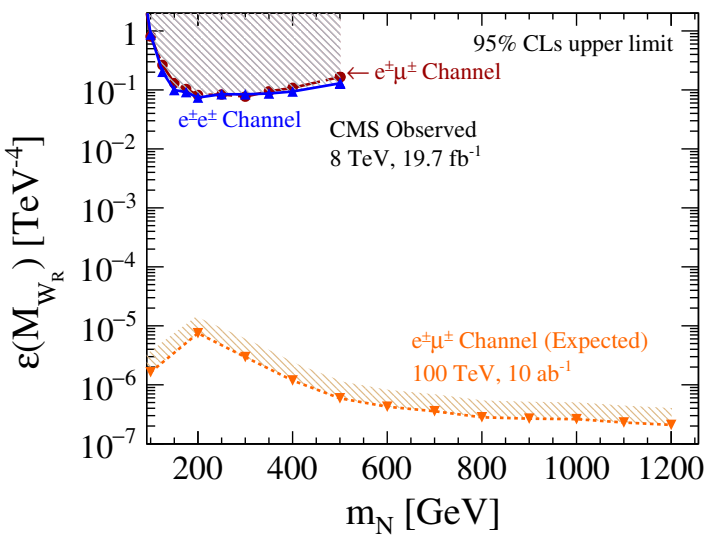

(b)

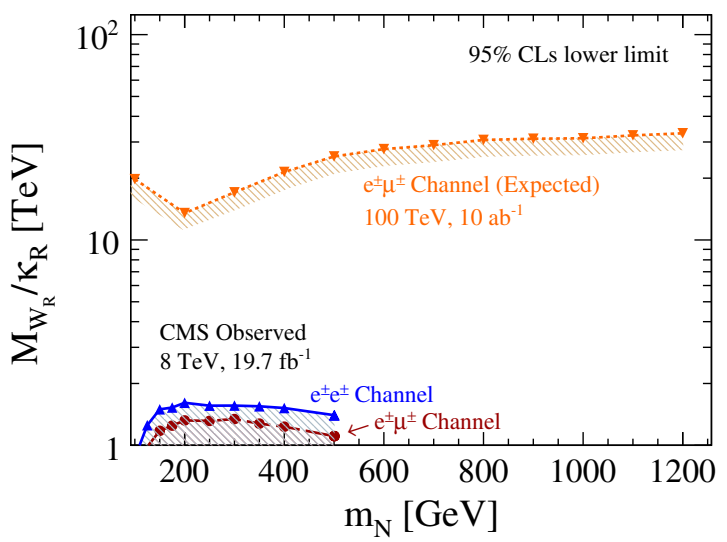

(d)

(dash-dot) and $e^{ \pm} e^{ \pm}$(solid-triangle) at $8 \mathrm{TeV}$ and $e \mu$ (dot-star) at 100 TeV.c, d Same as a, b, respectively, but for lower bounds on $\left(M_{W_{R}} / \kappa_{R}\right)$. All limits are obtained at $95 \% \mathrm{CL}_{s}$

eter space excluded by the ATLAS experiment at $8 \mathrm{TeV}$ with $20.3 \mathrm{fb}^{-1}$ in the $\mu \mu$ channel [13], along with our corresponding sensitivities for $\kappa_{R}=1$. For $m_{N} \approx 100-500 \mathrm{GeV}$, we find that the reinterpretation of CMS's DP limits are actually within $1.5 \times$ of present $M_{W_{R}}$ limits from resonant $W_{R}-N$ and dijet (not shown) searches [12,13,28,29]. However, for such low mass scales, the validity of this approach requires $\kappa_{R} \gg 1$. With $100 \mathrm{fb}^{-1}$ at $14 \mathrm{TeV}$, projected sensitivities are competitive with the $\mathscr{O}(5) \mathrm{TeV}$ reach from resonant searches using the full HL-LHC dataset $[16,19,36]$. With $1 \mathrm{ab}^{-1}$ at $14 \mathrm{TeV}$, and more so with $10 \mathrm{ab}^{-1}$ at $100 \mathrm{TeV}$, the DP channel can probe super heavy $v_{R}$ scales favored by low-energy probes [23-27]. These findings suggest searches for heavy Majorana neutrinos via off-shell $W_{R}$ may be of some usefulness at current and future collider experiments.

For completeness, upper limits on $\varepsilon^{\mu \mu}\left(M_{W_{R}}\right)$ are recast in terms of the NEFT operators in Eq. (8). Using Eqs. (27), (28), the lower bounds on $\left(\Lambda / \sqrt{\alpha_{V, S 3}}\right)$ are 


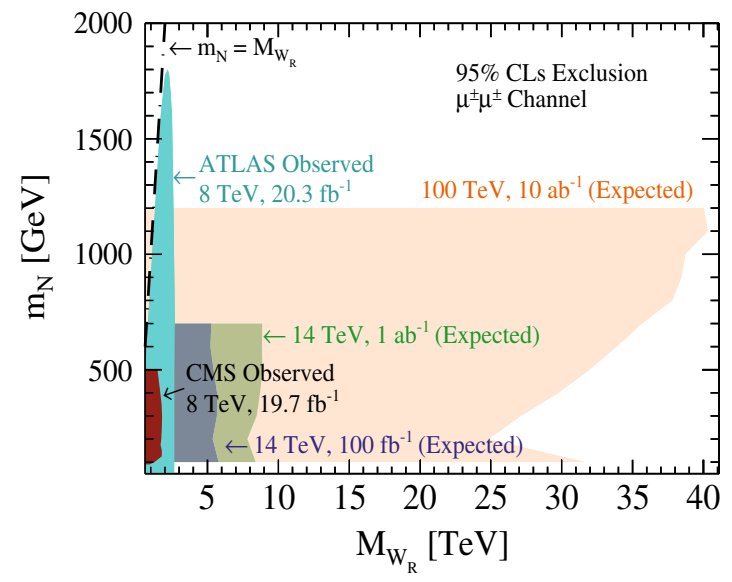

(a)

Fig. 3 a Observed and expected $95 \% \mathrm{CL}_{s}$ sensitivities to the $\left(M_{W_{R}}, m_{N}\right)$ parameter space $\left(\kappa_{R}=1\right)$ for various collider configurations via direct and indirect searches in the $\mu^{ \pm} \mu^{ \pm}$final state. b Observed

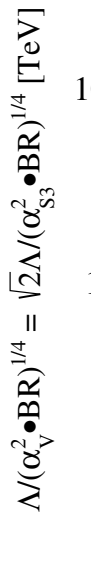

and expected $95 \% \mathrm{CL}_{s}$ sensitivities to the NEFT dimension-six operators $\mathscr{O}_{V}^{(6)}$ and $\mathscr{O}_{S 3}^{(6)}$ in the $\mu^{ \pm} \mu^{ \pm}$channel for the collider configurations in Fig. 2a

Table 1 Observed [41,42] and expected [43] 95\% $\mathrm{CL}_{s}$ sensitivities to $\varepsilon\left(M_{W_{R}}\right)$ and $\left(M_{W_{R}} / \kappa_{R}\right)$ in the LRSM as well as $\Lambda / \sqrt[4]{\alpha_{V}^{2} \mathrm{BR}}$ in NEFT assuming various $p p$ collider energies $(\sqrt{s})$ and integrated luminosity caches $(\mathscr{L})$

\begin{tabular}{|c|c|c|c|c|c|c|c|c|c|c|c|c|c|}
\hline \multirow{3}{*}{$\begin{array}{l}\sqrt{s}(\mathrm{TeV}) \\
\mathscr{L}\left(\mathrm{fb}^{-1}\right) \\
m_{N}(\mathrm{GeV})\end{array}$} & \multirow[b]{3}{*}{$\ell_{1}^{ \pm} \ell_{2}^{ \pm}$} & \multirow{2}{*}{$\begin{array}{l}\text { Obs. } \\
8 \\
19.7\end{array}$} & \multicolumn{3}{|l|}{ Exp. } & \multirow{2}{*}{$\begin{array}{l}\text { Obs. } \\
8 \\
19.7\end{array}$} & \multicolumn{3}{|c|}{ Exp. } & \multirow{2}{*}{$\begin{array}{l}\text { Obs. } \\
8 \\
19.7\end{array}$} & \multicolumn{3}{|l|}{ Exp. } \\
\hline & & & $\begin{array}{l}14 \\
100\end{array}$ & $\begin{array}{l}14 \\
10^{3}\end{array}$ & $\begin{array}{l}100 \\
10^{4}\end{array}$ & & $\begin{array}{l}14 \\
100\end{array}$ & $\begin{array}{l}14 \\
10^{3}\end{array}$ & $\begin{array}{l}100 \\
10^{4}\end{array}$ & & $\begin{array}{l}14 \\
100\end{array}$ & $\begin{array}{l}14 \\
10^{3}\end{array}$ & $\begin{array}{l}100 \\
10^{4}\end{array}$ \\
\hline & & \multicolumn{4}{|c|}{$\varepsilon\left(M_{W_{R}}\right)\left(\mathrm{TeV}^{-4}\right)$} & \multicolumn{4}{|c|}{$M_{W_{R}} / \kappa_{R}(\mathrm{TeV})$} & \multicolumn{4}{|c|}{$\Lambda / \sqrt[4]{\alpha_{V}^{2} \cdot \mathrm{BR}}(\mathrm{TeV})$} \\
\hline \multirow[t]{3}{*}{100} & $\mu \mu$ & $1.95 \times 10^{-1}$ & $6.45 \times 10^{-4}$ & $1.00 \times 10^{-4}$ & $4.96 \times 10^{-7}$ & 1.3 & 5.8 & 8.4 & 32 & 2.1 & 9.7 & 14 & 53 \\
\hline & $e \mu$ & $8.05 \times 10^{-1}$ & - & - & $1.64 \times 10^{-6}$ & 0.75 & - & - & 20 & 1.5 & - & - & 40 \\
\hline & $e e$ & $8.70 \times 10^{-1}$ & - & - & - & 0.87 & - & - & - & 1.5 & - & - & - \\
\hline \multirow[t]{3}{*}{200} & $\mu \mu$ & $5.44 \times 10^{-2}$ & $6.03 \times 10^{-4}$ & $1.34 \times 10^{-4}$ & $1.31 \times 10^{-6}$ & 1.7 & 5.4 & 7.8 & 25 & 2.9 & 9.0 & 13 & 42 \\
\hline & $e \mu$ & $8.19 \times 10^{-2}$ & - & - & $7.49 \times 10^{-6}$ & 1.3 & - & - & 14 & 2.6 & - & - & 27 \\
\hline & $e e$ & $7.42 \times 10^{-2}$ & - & - & - & 1.6 & - & - & - & 2.7 & - & - & - \\
\hline \multirow[t]{3}{*}{300} & $\mu \mu$ & $4.81 \times 10^{-2}$ & $6.84 \times 10^{-4}$ & $9.69 \times 10^{-5}$ & $9.22 \times 10^{-7}$ & 1.8 & 5.7 & 8.5 & 27 & 3.0 & 9.5 & 14 & 46 \\
\hline & $e \mu$ & $7.70 \times 10^{-2}$ & - & - & $2.95 \times 10^{-6}$ & 1.3 & - & - & 17 & 2.7 & - & - & 34 \\
\hline & $e e$ & $8.42 \times 10^{-2}$ & - & - & - & 1.6 & - & - & - & 2.6 & - & - & - \\
\hline \multirow[t]{3}{*}{500} & $\mu \mu$ & $1.06 \times 10^{-1}$ & $5.74 \times 10^{-4}$ & $8.04 \times 10^{-5}$ & $4.79 \times 10^{-7}$ & 1.5 & 5.4 & 8.9 & 32. & 2.5 & 9.1 & 15 & 54 \\
\hline & $e \mu$ & $1.66 \times 10^{-1}$ & - & - & $5.90 \times 10^{-7}$ & 1.1 & - & - & 26 & 2.2 & - & - & 51 \\
\hline & $e e$ & $1.29 \times 10^{-1}$ & - & - & - & 1.4 & - & - & - & 2.4 & - & - & - \\
\hline \multirow[t]{2}{*}{1200} & $\mu \mu$ & - & - & - & $1.95 \times 10^{-7}$ & - & - & - & 40 & - & - & - & 67 \\
\hline & $e \mu$ & - & - & - & $2.09 \times 10^{-7}$ & - & - & - & 33 & - & - & - & 66 \\
\hline
\end{tabular}

$\frac{\Lambda}{\sqrt[4]{\alpha_{V}^{2} \mathrm{BR}(N \rightarrow \mu X)}}>\sqrt[4]{\frac{4\left|Y_{\mu N}\right|^{2}}{\varepsilon_{\operatorname{Exp}(\mathrm{Obs})}^{\mu \mu}\left(M_{W_{R}}\right)}}$,

$\frac{\Lambda}{\sqrt[4]{\alpha_{S 3}^{2} \mathrm{BR}(N \rightarrow \mu X)}}>\sqrt[4]{\frac{\left|Y_{\mu N}\right|^{2}}{\varepsilon_{\operatorname{Exp}(\mathrm{Obs})}^{\mu \mu}\left(M_{W_{R}}\right)}}$.

As a function of $m_{N}$, the observed and expected sensitivities to $\mathscr{O}_{V}$ for the several configurations in Fig. 2a and mixing choice in Eq. (40) are shown in Fig. 3b. Over the respective ranges of $m_{N}$, they span approximately

$$
\begin{aligned}
& \mathscr{L}_{19.7 \mathrm{fb}^{-1}}^{8 \mathrm{TeV}}: \frac{\Lambda}{\sqrt[4]{\alpha_{V}^{2} \mathrm{BR}(N \rightarrow \mu X)}}>2.1-3.0 \mathrm{TeV}, \\
& \mathscr{L}_{100 \mathrm{fb}^{-1}}^{14 \mathrm{TeV}}: \frac{\Lambda}{\sqrt[4]{\alpha_{V}^{2} \mathrm{BR}(N \rightarrow \mu X)}}>8.7-9.7 \mathrm{TeV},
\end{aligned}
$$




$$
\begin{aligned}
& \mathscr{L}_{1 \mathrm{ab}^{-1}}^{14 \mathrm{TeV}}: \frac{\Lambda}{\sqrt[4]{\alpha_{V}^{2} \mathrm{BR}(N \rightarrow \mu X)}}>13-15 \mathrm{TeV}, \\
& \mathscr{L}_{10 \mathrm{ab}^{-1}}^{100 \mathrm{TeV}}: \frac{\Lambda}{\sqrt[4]{\alpha_{V}^{2} \mathrm{BR}(N \rightarrow \mu X)}}>42-68 \mathrm{TeV} .
\end{aligned}
$$

We summarize our reported findings in Table 1.

\section{Summary and conclusion}

While the LRSM naturally addresses shortcomings of the $\mathrm{SM}$, it is not guaranteed its entire particle spectrum lies within the kinematic reach of the LHC or a future $100 \mathrm{TeV}$ VLHC. Indeed, low-energy probes favor the LR breaking scale to be above the LHC's threshold [22-27].

In this context, we argue that when LRSM gauge bosons are too heavy to be produced resonantly, on-shell production of sub-TeV Majorana neutrinos via the process $p p \rightarrow$ $W_{R}^{*} \rightarrow N \ell^{ \pm} \rightarrow \ell^{ \pm} \ell^{ \pm}+n j$ is still possible when mediated by far off-shell $W_{R}$. In this regime, the process' mass scale and topology are identical to the direct production (DP) process $p p \rightarrow W_{\mathrm{SM}}^{*} \rightarrow N \ell^{ \pm} \rightarrow \ell^{ \pm} \ell^{ \pm}+n j$. Subsequently, searches for DP of heavy Majorana neutrinos can be translated into searches for LR symmetry.

We have recast current $[12,13]$ and projected $[36,43]$ sensitivities to the DP process at $p p$ colliders into observed and expected sensitivities for the LRSM, in the heavy $M_{W_{R}}$ limit. We find the following:

1. At the $8 \mathrm{TeV} \mathrm{LHC}$, for $m_{N}=100-500 \mathrm{GeV}$ and rightleft coupling ratio $\kappa_{R}=g_{R} / g_{L}$, searches have excluded at $95 \% \mathrm{CL}_{s}\left(M_{W_{R}} / \kappa_{R}\right)<0.7-1.8 \mathrm{TeV}$. For $m_{N} \gtrsim 200$ $\mathrm{GeV}$, this is within $1.5 \times$ of searches for resonant $W_{R}$ and $W_{R}-N$ production.

2. At $14 \mathrm{TeV}$ with $100 \mathrm{fb}^{-1}\left(1 \mathrm{ab}^{-1}\right)$, one can exclude at $95 \% \mathrm{CL}_{s}\left(M_{W_{R}} / \kappa_{R}\right)<5.2-5.8(7.8-8.9) \mathrm{TeV}$ for $m_{N}=100-700 \mathrm{GeV}$, well beyond the $\mathscr{O}(5) \mathrm{TeV}$ anticipated reach of resonant $W_{R}$ searches.

3. At $100 \mathrm{TeV}$ with $10 \mathrm{ab}^{-1}$, one can probe $\left(M_{W_{R}} / \kappa_{R}\right)<14$ $-40 \mathrm{TeV}$ at $95 \% \mathrm{CL}_{s}$ for $m_{N}=100-1200 \mathrm{GeV}$, thereby greatly complementing low-energy probes of $\mathscr{O}(10) \mathrm{TeV}$ $v_{R}$.

4. In terms of an Effective Field Theory featuring heavy neutrinos, we find limits on mass/coupling scales for gauge invariant, dimension-six operators comparable to the aforementioned limits in the LRSM.

Acknowledgements Peter Ballett, Lydia Brenner, Luca Di Luzio, Silvia Pascoli, Carlos Fibo Tamarit, and Cedric Weiland are thanked for discussions. This work was funded in part by the UK Science and Technology Facilities Council, and the European Union's Horizon 2020 research and innovation programme under the Marie Sklodowska-Curie Grant agreement 674896 (Elusives ITN).
Open Access This article is distributed under the terms of the Creative Commons Attribution 4.0 International License (http://creativecomm ons.org/licenses/by/4.0/), which permits unrestricted use, distribution, and reproduction in any medium, provided you give appropriate credit to the original author(s) and the source, provide a link to the Creative Commons license, and indicate if changes were made.

Funded by $\mathrm{SCOAP}^{3}$.

\section{References}

1. J. C. Pati and A. Salam, Phys. Rev. D 10, 275 (1974). doi:10. 1103/PhysRevD.10.275. doi:10.1103/PhysRevD.11.703.2 [Erratum: Phys. Rev. D 11, 703 (1975)]

2. R.N. Mohapatra, J.C. Pati, Phys. Rev. D 11, 566 (1975). doi:10. 1103/PhysRevD.11.566

3. R.N. Mohapatra, J.C. Pati, Phys. Rev. D 11, 2558 (1975). doi:10. 1103/PhysRevD.11.2558

4. G. Senjanovic, R.N. Mohapatra, Phys. Rev. D 12, 1502 (1975). doi:10.1103/PhysRevD.12.1502

5. G. Senjanovic, Nucl. Phys. B 153, 334 (1979). doi:10.1016/ 0550-3213(79)90604-7

6. N. Arkani-Hamed, T. Han, M. Mangano, L.T. Wang, Phys. Rep. 652, 1 (2016). doi:10.1016/j.physrep.2016.07.004. arXiv:1511.06495 [hep-ph]

7. T. Golling et al., Phys. Rept. (2016, under review). arXiv:1606.00947 [hep-ph]

8. M.C. Chen, J. Huang, Mod. Phys. Lett. A 26, 1147 (2011). doi:10. 1142/S0217732311035985. arXiv:1105.3188 [hep-ph]

9. R.N. Mohapatra, PoS Neutel 2013, 050 (2013)

10. R.N. Mohapatra, Nucl. Phys. B 908, 423 (2016). doi:10.1016/j. nuclphysb.2016.03.006

11. G. Senjanovic, Mod. Phys. Lett. A 32(04), 1730004 (2017). doi:10. 1142/S021773231730004X. arXiv:1610.04209 [hep-ph]

12. V. Khachatryan et al., CMS Collaboration, Eur. Phys. J. C 74(11), 3149 (2014). doi:10.1140/epjc/s10052-014-3149-z. arXiv: 1407.3683 [hep-ex]

13. G. Aad et al., ATLAS Collaboration, JHEP 1507, 162 (2015). doi:10.1007/JHEP07(2015)162. arXiv:1506.06020 [hep-ex]

14. W.Y. Keung, G. Senjanovic, Phys. Rev. Lett. 50, 1427 (1983). doi:10.1103/PhysRevLett.50.1427

15. D. Binosi, L. Theussl, JaxoDraw: a Graphical user interface for drawing Feynman diagrams. Comput. Phys. Commun. 161, 76 (2004). arXiv:hep-ph/0309015

16. A. Ferrari, J. Collot, M.L. Andrieux, B. Belhorma, P. de Saintignon, J.Y. Hostachy, P. Martin, M. Wielers, Phys. Rev. D 62, 013001 (2000). doi:10.1103/PhysRevD.62.013001

17. A. Maiezza, M. Nemevšek F. Nesti, Phys. Rev. Lett. 115, 081802 (2015). doi:10.1103/PhysRevLett.115.081802. arXiv:1503.06834 [hep-ph]

18. J. Gluza, T. Jelinski, R. Szafron, Phys. Rev. D 93(11), 113017 (2016). doi:10.1103/PhysRevD.93.113017. arXiv:1604.01388 [hep-ph]

19. M. Mitra, R. Ruiz, D.J. Scott, M. Spannowsky, Phys. Rev. D 94(9), 095016 (2016). doi:10.1103/PhysRevD.94.095016. arXiv:1607.03504 [hep-ph]

20. O. Mattelaer, M. Mitra, R. Ruiz, Phys. Rev. D (2016, under review). arXiv: 1610.08985 [hep-ph]

21. E.H. Simmons, New gauge interactions and single top quark production. Phys. Rev. D 55, 5494 (1997). doi:10.1103/PhysRevD.55. 5494. arXiv:hep-ph/9612402

22. J. Chakrabortty, J. Gluza, R. Sevillano, R. Szafron, JHEP 1207, 038 (2012). doi:10.1007/JHEP07(2012)038. arXiv:1204.0736 [hep$\mathrm{ph}]$ 
23. S. Bertolini, A. Maiezza, F. Nesti, Phys. Rev. D 89(9), 095028 (2014). doi:10.1103/PhysRevD.89.095028. arXiv:1403.7112 [hep-ph]

24. A. Maiezza, M. NemevÅąek, Phys. Rev. D 90(9), 095002 (2014). doi:10.1103/PhysRevD.90.095002. arXiv:1407.3678 [hep-ph]

25. A. Maiezza, M. NemevÅąek, F. Nesti, Phys. Rev. D 94(3), 035008 (2016). doi:10.1103/PhysRevD.94.035008. arXiv:1603.00360 [hep-ph]

26. Y. Zhang, H. An, X. Ji, R.N. Mohapatra, Phys. Rev. D 76, 091301 (2007). doi:10.1103/PhysRevD.76.091301. arXiv:0704.1662 [hep-ph]

27. Y. Zhang, H. An, X. Ji, R.N. Mohapatra, Nucl. Phys. B 802, 247 (2008). doi:10.1016/j.nuclphysb.2008.05.019. arXiv:0712.4218 [hep-ph]

28. V. Khachatryan et al., CMS Collaboration, Phys. Rev. Lett. 116(7), 071801 (2016). doi:10.1103/PhysRevLett.116.071801. arXiv:1512.01224 [hep-ex]

29. G. Aad et al., ATLAS Collaboration, Phys. Lett. B 754, 302 (2016). doi:10.1016/j.physletb.2016.01.032. arXiv:1512.01530 [hep-ex]

30. G. Aad et al., ATLAS Collaboration, Eur. Phys. J. C 72, 2056 (2012). doi:10.1140/epjc/s10052-012-2056-4. arXiv:1203.5420 [hep-ex]

31. D.A. Dicus, D.D. Karatas, P. Roy, Phys. Rev. D 44, 2033 (1991). doi:10.1103/PhysRevD.44.2033

32. A. Pilaftsis, Z. Phys. C 55, 275 (1992). doi:10.1007/BF01482590. arXiv:hep-ph/9901206

33. A. Datta, M. Guchait, A. Pilaftsis, Phys. Rev. D 50, 3195 (1994). doi:10.1103/PhysRevD.50.3195. arXiv:hep-ph/9311257

34. T. Han, B. Zhang, Phys. Rev. Lett. 97, 171804 (2006). doi:10.1103/ PhysRevLett.97.171804. arXiv:hep-ph/0604064

35. A. Atre, T. Han, S. Pascoli, B. Zhang, JHEP 0905, 030 (2009). doi:10.1088/1126-6708/2009/05/030. arXiv:0901.3589 [hep-ph]

36. T. Han, I. Lewis, R. Ruiz, Z.G. Si, Phys. Rev. D 87(3), 035011 (2013). doi:10.1103/PhysRevD.87.035011. arXiv:1211.6447 [hep-ph]

37. C.Y. Chen, P.S.B. Dev, R.N. Mohapatra, Phys. Rev. D 88, 033014 (2013). doi:10.1103/PhysRevD.88.033014. arXiv:1306.2342 [hep-ph]

38. P.S.B. Dev, D. Kim, R.N. Mohapatra, JHEP 1601, 118 (2016). doi:10.1007/JHEP01(2016)118. arXiv:1510.04328 [hep-ph]

39. M. Nemevsek, G. Senjanovic, V. Tello, Phys. Rev. Lett. 110(15), 151802 (2013). doi:10.1103/PhysRevLett.110.151802. arXiv:1211.2837 [hep-ph]

40. G. Senjanovic, V. Tello, arXiv:1612.05503 [hep-ph]

41. V. Khachatryan et al., CMS Collaboration, Phys. Lett. B 748, 144 (2015). doi:10.1016/j.physletb.2015.06.070. arXiv:1501.05566 [hep-ex]

42. V. Khachatryan et al., CMS Collaboration, JHEP 1604, 169 (2016). doi:10.1007/JHEP04(2016)169. arXiv:1603.02248 [hep-ex]

43. D. Alva, T. Han, R. Ruiz, JHEP 1502, 072 (2015). doi:10.1007/ JHEP02(2015)072. arXiv:1411.7305 [hep-ph]

44. F. del Aguila, S. Bar-Shalom, A. Soni, J. Wudka, Phys. Lett. B 670, 399 (2009). doi:10.1016/j.physletb.2008.11.031. arXiv:0806.0876 [hep-ph]

45. A. Maiezza, M. Nemevsek, F. Nesti, G. Senjanovic, Phys. Rev. D 82, 055022 (2010). doi:10.1103/PhysRevD.82.055022. arXiv: 1005.5160 [hep-ph]

46. G. Senjanovi, V. Tello, Phys. Rev. Lett. 114(7), 071801 (2015). doi:10.1103/PhysRevLett.114.071801. arXiv:1408.3835 [hep-ph]
47. G. Senjanovic, V. Tello, Phys. Rev. D 94(9), 095023 (2016). doi:10. 1103/PhysRevD.94.095023. arXiv:1502.05704 [hep-ph]

48. A. Aparici, K. Kim, A. Santamaria, J. Wudka, Phys. Rev. D 80, 013010 (2009). doi:10.1103/PhysRevD.80.013010. arXiv:0904.3244 [hep-ph]

49. S. Bhattacharya and J. Wudka, Phys. Rev. D 94, no. 5, 055022 (2016). doi:10.1103/PhysRevD.94.055022, doi:10. 1103/PhysRevD.95.039904. arXiv:1505.05264 [hep-ph] [Erratum: Phys. Rev. D 95(3), 039904 (2017)]

50. W. Buchmuller, D. Wyler, Nucl. Phys. B 268, 621 (1986). doi:10. 1016/0550-3213(86)90262-2

51. B. Grzadkowski, M. Iskrzynski, M. Misiak, J. Rosiek, JHEP 1010, 085 (2010). doi:10.1007/JHEP10(2010)085. arXiv:1008.4884 [hep-ph]

52. E. Ma, Phys. Rev. Lett. 81, 1171 (1998). doi:10.1103/PhysRevLett. 81.1171. arXiv:hep-ph/9805219

53. J. Kersten, A.Y. Smirnov, Phys. Rev. D 76, 073005 (2007). doi:10. 1103/PhysRevD.76.073005. arXiv:0705.3221 [hep-ph]

54. P. Minkowski, Phys. Lett. B 67, 421 (1977)

55. T. Yanagida, Conf. Proc. C 7902131, 95 (1979)

56. M. Gell-Mann, P. Ramond, R. Slansky, Conf. Proc. C 790927, 315 (1979)

57. R.N. Mohapatra, G. Senjanovic, Phys. Rev. Lett. 44, 912 (1980)

58. B. Henning, X. Lu, T. Melia, H. Murayama (2015). arXiv: 1512.03433 [hep-ph]

59. A. Kobach, Phys. Lett. B 758, 455 (2016). doi:10.1016/j.physletb. 2016.05.050. arXiv:1604.05726 [hep-ph]

60. S. Gopalakrishna, T. Han, I. Lewis, Z.G. Si, Y.F. Zhou, Phys. Rev. D 82, 115020 (2010). doi:10.1103/PhysRevD.82.115020. arXiv: 1008.3508 [hep-ph]

61. B.W. Harris, J.F. Owens, Phys. Rev. D 65, 094032 (2002). doi:10. 1103/PhysRevD.65.094032. arXiv:hep-ph/0102128

62. R. Ruiz, JHEP 1512, 165 (2015). doi:10.1007/JHEP12(2015)165. arXiv:1509.05416 [hep-ph]

63. N.D. Christensen, C. Duhr, FeynRules-Feynman rules made easy. Comput. Phys. Commun. 180, 1614 (2009). doi:10.1016/j.cpc. 2009.02.018. arXiv:0806.4194 [hep-ph]

64. A. Alloul, N.D. Christensen, C. Degrande, C. Duhr, B. Fuks, FeynRules 2.0-a complete toolbox for tree-level phenomenology. Comput. Phys. Commun. 185, 2250 (2014). doi:10.1016/j. cpc.2014.04.012. arXiv:1310.1921 [hep-ph]

65. C. Degrande, Comput. Phys. Commun. 197, 239 (2015). doi:10. 1016/j.cpc.2015.08.015. arXiv:1406.3030 [hep-ph]

66. J. Alwall et al., The automated computation of tree-level and nextto-leading order differential cross sections, and their matching to parton shower simulations. JHEP 1407, 079 (2014). doi:10.1007/ JHEP07(2014)079. arXiv:1405.0301 [hep-ph]

67. A.L. Read, J. Phys. G 28, 2693 (2002). doi:10.1088/0954-3899/ $28 / 10 / 313$

68. T. Junk, Nucl. Instrum. Meth. A 434, 435 (1999). doi:10.1016/ S0168-9002(99)00498-2. arXiv:hep-ex/9902006

69. ATLAS Collaboration, ATL-PHYS-PUB-2011-011, ATL-COMPHYS-2011-818, CMS-NOTE-2011-005 (2011)

70. I. Hinchliffe, A. Kotwal, M.L. Mangano, C. Quigg, L.T. Wang, Int. J. Mod. Phys. A 30(23), 1544002 (2015). doi:10.1142/ S0217751X15440029. arXiv:1504.06108 [hep-ph] 